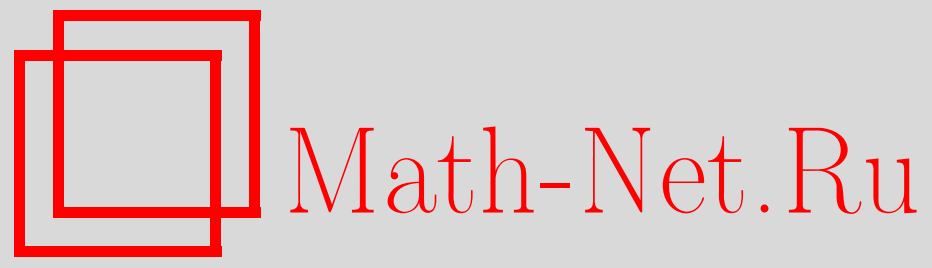

Л. В. Розовский, О точной асимптотике в слабом законе больших чисел для сумм независимых случайных величин с общей функцией распределения из области притяжения устойчивого закона. II, Теория вероятн. и ее примен., 2004, том 49, выпуск 4, 803-813

DOI: https://doi.org/10.4213/tvp198

Использование Общероссийского математического портала Math-Net.Ru подразумевает, что вы прочитали и согласны с пользовательским соглашением

http://www . mathnet.ru/rus/agreement

Параметры загрузки:

IP : 3.91 .87 .62

26 апреля 2023 г., 13:07:07

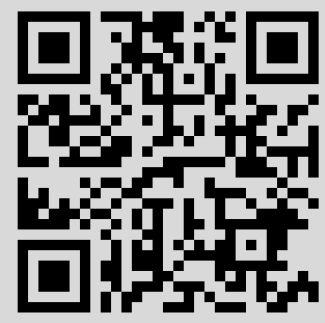




\section{О ТОЧНОЙ АСИМПТОТИКЕ В СЛАБОМ ЗАКОНЕ БОЛЬШИХ ЧИСЕЛ ДЛЯ СУММ НЕЗАВИСИМЫХ СЛУЧАЙНЫХ ВЕЛИЧИН С ОБЩЕЙ ФУНКЦИЕЙ РАСПРЕДЕЛЕНИЯ ИЗ ОБЛАСТИ ПРИТЯЖЕНИЯ УСТОЙЧИВОГО ЗАКОНА. II $^{1)}$}

Рассмотрим независимые одинаково распределенные случайные величины $X_{1}, X_{2}, \ldots$ такие, что

$$
U_{n}=\frac{1}{B_{n}} S_{n}-n a_{n} \longrightarrow \xi_{\alpha} \quad \text { по вероятности при } n \rightarrow \infty,
$$

где $S_{n}=X_{1}+\cdots+X_{n}$ и $B_{n}>0, a_{n}$ - некоторые числа $(n \geqslant 1)$, а случайная величина $\xi_{\alpha}$ имеет некоторое устойчивое распределение с характеристическим показателем $\alpha \in[1,2]$, причем при $\alpha \neq 2$ предполагается, что правый хвост распределения $\xi_{\alpha}$ убывает быстрее его левого хвоста.

Целью работы является нахождение условий, при которых

$$
\sum_{n} f_{n} \mathbf{P}\left\{U_{n} \geqslant \varepsilon \varphi_{n}\right\} \sim \sum_{n} f_{n} \mathbf{P}\left\{\xi_{\alpha} \geqslant \varepsilon \varphi_{n}\right\}, \quad \varepsilon \searrow 0,
$$

где $\varphi_{n}$ - положительная последовательность, монотонно растушая к бесконечности и удовлетворяюшая некоторым дополнительным ограничениям, а $f_{n}$ - неотрицательная последовательность такая, что $\sum_{n} f_{n}=\infty$.

Ключевые слова и фразы: независимые случайные величины, закон больших чисел, устойчивый закон.

1. Введение. Результаты. Рассмотрим независимые случайные величины $X, X_{1}, X_{2}, \ldots$ с общей функцией распределения $F$. Положим $S_{n}=X_{1}+\cdots+X_{n}$ $(n \geqslant 1)$.

Будем предполагать, что $F$ принадлежит области притяжения некоторого невырожденного устойчивого распределения $F_{\alpha}$ с характеристическим показателем $\alpha$, т.е. при подходящих $a_{n}$ и $B_{n}>0$ выполняется условие

$$
U_{n}=\frac{1}{B_{n}} S_{n}-n a_{n} \longrightarrow \xi_{\alpha} \quad \text { по вероятности при } n \rightarrow \infty,
$$

где случайная величина $\xi_{\alpha}$ имеет распределение $F_{\alpha}$.

Так же, как в [8], нас интересуют условия, при которых

$$
S(\varepsilon)=\sum_{n \geqslant 1} f_{n} \mathbf{P}\left\{U_{n} \geqslant \varepsilon \varphi_{n}\right\} \sim \sum_{n \geqslant 1} f_{n} \mathbf{P}\left\{\xi_{\alpha} \geqslant \varepsilon \varphi_{n}\right\}, \quad \varepsilon \searrow 0,
$$

где числовые последовательности $f_{n}, \varphi_{n}$ удовлетворяют условиям

$$
f_{n} \geqslant 0, \quad \sum_{n \geqslant 1} f_{n}=\infty, \quad \varphi_{n} \nearrow \infty .
$$

Однако, в отличие от [8], будет рассмотрен характеристический показатель $\alpha=2$ (когда $F_{\alpha}$ является нормальным распределением), а также случай $1 \leqslant \alpha<2$ в предположении, что правый хвост распределения $F_{\alpha}$ убывает быстрее левого:

$$
l_{\alpha}=\lim _{x \rightarrow+\infty} \frac{1-F_{\alpha}(x)}{F_{\alpha}(-x)}=0 .
$$

* С.-Петербургская химико-фармацевтическая академия, кафедра высшей математики, ул. проф. Попова, 14, 197376 С.-Петербург, Россия; e-mail: l_rozovski@mail.ru

1) Работа выполнена при поддержке гранта Министерства образования № Е02-1.0-219, гранта РФФИ № 02-01-01099 и гранта НШ № 2258.2003.1. 
Принимая во внимание то, что случай $l_{\alpha}>0(0<\alpha<2)$ изучался в [8], и учитывая, что предел $l_{\alpha}$ всегда сушествует, а также то, что $F_{\alpha}(0)=1$ без потери общности, если $l_{\alpha}=0(0<\alpha<1)$, можно считать, что настояшая работа и [8] охватывают весь допустимый набор предельных устойчивых распределений $F_{\alpha}$, при которых имеет смысл изучать соотношение (1.2).

Введем обозначения. Положим

$$
\bar{F}(x)=1-F(x), \quad \bar{F}_{\alpha}(x)=1-F_{\alpha}(x), \quad \psi_{\alpha}(\varepsilon)=\sum_{n \geqslant 1} f_{n} \bar{F}_{\alpha}\left(\varepsilon \varphi_{n}\right) ;
$$

кроме того, для произвольной последовательности $\lambda_{1}, \lambda_{2}, \ldots$ будем использовать обозначение $\Delta \lambda_{m}=\lambda_{m+1}-\lambda_{m}$. в п. 2 .

Сформулируем основной результат статьи, доказательство которого содержится

Предложение 1. Предположим, что при некотором $\alpha \in[1,2]$ выполняется условие (1.1), причем

$$
\lim _{x \rightarrow+\infty} \frac{\bar{F}_{\alpha}(x)}{F_{\alpha}(-x)}=0 \quad \text { npu } \quad \alpha \neq 2 .
$$

Пусть $f_{n}$ и $\varphi_{n}$ удовлетворяют условию (1.3), а последовательность $b_{n}=B_{n} \varphi_{n}$ не убицвает. Если

$$
\limsup _{k \rightarrow \infty} \frac{\varphi_{k+1}}{\varphi_{k}}<\infty, \quad \limsup _{k \rightarrow \infty} \frac{\varphi_{k}^{\delta} \sum_{n>k} f_{n} \varphi_{n}^{-\delta}}{\sum_{n=1}^{k} f_{n}}<\infty \quad(\exists \delta>0)
$$

$u$

$$
\limsup _{k \rightarrow \infty} \frac{\Delta b_{k}}{b_{k}} \frac{b_{k}^{l}}{f_{k}} \sum_{n>k} f_{n} b_{n}^{-l}<\infty \quad(\exists l>0),
$$

mo

$$
\sum_{n \geqslant 1} f_{n} \mathbf{P}\left\{U_{n} \geqslant \varepsilon \varphi_{n}\right\} \sim \psi_{\alpha}(\varepsilon), \quad \varepsilon \searrow 0
$$

тогда и только тогда, когда

$$
\lim _{\varepsilon \searrow 0} \frac{1}{\psi_{\alpha}(\varepsilon)} \sum_{n: \varepsilon \varphi_{n} \geqslant 1} n f_{n} \bar{F}\left(\varepsilon b_{n}\right)=0
$$

Заметим, что условие (1.6) выполняется, если

$$
\max _{n>k} \frac{f_{n} b_{n}^{-l}}{\Delta b_{n}}=O(1) \frac{f_{k} b_{k}^{-l}}{\Delta b_{k}}, \quad k \rightarrow \infty \quad(\exists l>0) .
$$

Приведем одно полезное следствие предложения 1 .

Теорема 1. Пусть при некотором $\alpha \in[1,2]$ выполняются условия (1.1) $и$ (1.4). Пусть последовательности $\varphi_{n}$ u $b_{n}=B_{n} \varphi_{n}$ монотонно растут $\kappa$ бесконечности $u$

$$
\varphi_{n} \sim \varphi_{n+1}, \quad n \rightarrow \infty
$$

Пусть также

$$
f_{n} \sim \int_{\varphi_{n}}^{\varphi_{n+1}} g(u) \frac{d u}{u}, \quad n \rightarrow \infty,
$$

где положительная функиия $g(u)$ удовлетворяет условиям

$$
\int_{1}^{\infty} g(u) \frac{d u}{u}=\infty
$$

$\frac{g(u)}{u^{\delta}} \quad$ ббьвает при некотором $\delta>0 \quad$ ивсех достаточно больиих $\quad$ и.

Eсли

$$
\max _{n>k} \frac{\Delta \varphi_{n}}{\varphi_{n}} \frac{b_{n}}{\Delta b_{n}} b_{n}^{-\gamma}=O(1) \frac{\Delta \varphi_{k}}{\varphi_{k}} \frac{b_{k}}{\Delta b_{k}} b_{k}^{-\gamma}, \quad k \rightarrow \infty \quad(\exists \gamma>0)
$$


то соотношения

$$
\sum_{n \geqslant 1} f_{n} \mathbf{P}\left\{U_{n} \geqslant \varepsilon \varphi_{n}\right\} \sim \widetilde{\psi}_{\alpha}(\varepsilon)=\int_{1}^{\infty} g(u) \bar{F}_{\alpha}(\varepsilon u) \frac{d u}{u}, \quad \varepsilon \searrow 0,
$$

$u$

$$
\lim _{\varepsilon \searrow 0} \frac{1}{\widetilde{\psi}_{\alpha}(\dot{\varepsilon})} \sum_{n: \varepsilon \varphi_{n} \geqslant 1} n f_{n} \bar{F}\left(\varepsilon b_{n}\right)=0
$$

равносильнь.

3 а м е ч а н и е 1. В условиях (1.9)-(1.11) вместо функции $g(u)$ можно рассматривать любую эквивалентную ей (при $u \rightarrow \infty)$ функцию.

3 а м е ч а н и е 2. Если (1.11) выполняется при $\delta<\alpha$, то в условиях теоремы 1 соотношение (1.14) (следовательно и (1.13)) всегда имеет место.

3 а м е ч а н и е 3 . Пусть положительная функция $g(u)$ правильно меняется на бесконечности с показателем $\rho \geqslant 0$. Тогда при $\varepsilon \searrow 0$

$$
\begin{array}{ll}
\tilde{\psi}_{\alpha}(\varepsilon) \sim g\left(\frac{1}{\varepsilon}\right) \rho^{-1} \mathbf{E}\left(\xi_{\alpha}\right)_{+}^{\rho}, & \rho>0, \\
\tilde{\psi}_{\alpha}(\varepsilon) \sim \bar{F}_{\alpha}(0) \int_{1}^{1 / \varepsilon} g(u) \frac{d u}{u}, & \rho=0
\end{array}
$$

(в (1.15) и всюду в дальнейшем используется обозначение $\left.x_{+}=\max (0, x)\right)$.

Следует сказать, что во всех типичных случаях условие (1.12) выполняется. В частности, это условие справедливо, если

$$
0<\liminf n \frac{\Delta B_{n}}{B_{n}} \leqslant \lim \sup n \frac{\Delta B_{n}}{B_{n}}<\infty
$$

И

$$
\max _{k>n} \frac{\gamma_{k}}{1+\gamma_{k}}\left(k \varphi_{k}\right)^{-\delta}=O(1) \frac{\gamma_{n}}{1+\gamma_{n}}\left(n \varphi_{n}\right)^{-\delta}, \quad n \rightarrow \infty \quad(\exists \delta>0),
$$

где $\gamma_{n}=n \Delta \varphi_{n} / \varphi_{n}$.

По поводу условий $(1.17),(1.18)$ заметим, что первое из них имеет место во всех разумных ситуациях - обычно

$$
\lim n \frac{\Delta B_{n}}{B_{n}}=\frac{1}{\alpha}
$$

а второе выполняется, если $\varphi_{n} / n^{\gamma} \nearrow(\exists \gamma>0)$ или если $\varphi_{n} / n^{\gamma} \searrow(\exists \gamma>0)$ и

$$
\max _{k>n} \gamma_{k} k^{-\delta}=O(1) \gamma_{n} n^{-\delta}, \quad n \rightarrow \infty \quad(\exists \delta>0) .
$$

Отметим также, что условие (1.19) равносильно условию

$$
B_{n}=n^{1 / \alpha} h_{n}, \quad \text { где } \quad h_{n}>0, \quad n \frac{\Delta h_{n}}{h_{n}} \rightarrow 0 .
$$

В качестве одной из иллюстраций вышесказанного приведем следующий результат.

Теорема 2. Пусть при некотором $\alpha \in[1,2]$ выполняются условия (1.1) и (1.17). Пусть, кроме того, положительная функиия $g(u)$ правильно меняется на бесконечности с показателем $0 \leqslant \rho<\alpha$ и при $\rho=0$ удовлетворяет условию (1.10), а монотонно растущая $\kappa$ бесконечности последовательность $\varphi_{n}$ удовлетворяет условиям (1.8) и (1.18). Тогда приє \0

$$
\sum_{n} g\left(\varphi_{n}\right) \frac{\Delta \varphi_{n}}{\varphi_{n}} \mathbf{P}\left\{U_{n} \geqslant \varepsilon \varphi_{n}\right\} \sim \begin{cases}g\left(\frac{1}{\varepsilon}\right) \rho^{-1} \mathbf{E}\left(\xi_{\alpha}\right)_{+}^{\rho}, & \rho>0, \\ \bar{F}_{\alpha}(0) \int_{1}^{1 / \varepsilon} g(u) \frac{d u}{u}, & \rho=0 .\end{cases}
$$

Теорема 2 при выполнении условия (1.4) является прямым следствием теоремы 1 и замечаний 1-3, а в случае его нарушения вытекает из [8, следствие 2]. 
Следствие 1. Предположим, что при некотором $\alpha \in(1,2]$ выполняются условия (1.21) $u$

$$
\frac{S_{n}}{B_{n}} \longrightarrow \xi_{\alpha} \quad \text { по вероятности при } \quad n \rightarrow \infty .
$$

Пусть $b_{n}=n^{\gamma+1 / \alpha} l_{n}, \gamma>0$, где положительная последовательность $\left(l_{n}\right)$ удовлетворяет условию $n \Delta l_{n} / l_{n} \rightarrow 0$. Если $\rho \in[0, \alpha)$, то при $\varepsilon \searrow 0$

$$
\sum_{n} n^{\gamma \rho-1}\left(\frac{l_{n}}{h_{n}}\right)^{\rho} \mathbf{P}\left\{S_{n} \geqslant \varepsilon b_{n}\right\} \sim \frac{1}{\gamma} \begin{cases}\rho^{-1} \varepsilon^{-\rho} \mathbf{E}\left(\xi_{\alpha}\right)_{+}^{\rho}, & \rho>0, \\ \bar{F}_{\alpha}(0) \ln \frac{1}{\varepsilon}, & \rho=0 .\end{cases}
$$

Заметим, что следствие 1 при $\gamma \rho=r / p-1$ и $p=\alpha /(\alpha \gamma+1)$ является правосторонней версией (и уточнением) результатов работ [2] и [7].

Обозначим через $\ln _{j} n(j=1,2, \ldots)$-кратный натуральный логарифм $n$ (т.е. $\ln _{1} n=\ln n, \ln 2 n=\ln \ln n$ и т.д.).

Следствие 2. Пусть $\gamma>0,0 \leqslant \rho<\alpha$. Если при некотором $\alpha \in(1,2]$ выполнены условия (1.19) и (1.22), то при $\varepsilon \searrow 0$

$$
\sum_{n: \ln _{k} n \geqslant 1}\left(\ln _{k} n\right)^{\gamma \rho} \frac{1}{n \prod_{j=1}^{k} \ln _{j} n} \mathbf{P}\left\{S_{n} \geqslant \varepsilon B_{n}\left(\ln _{k} n\right)^{\gamma}\right\} \sim \frac{1}{\gamma} \begin{cases}\rho^{-1} \varepsilon^{-\rho} \mathbf{E}\left(\xi_{\alpha}\right)_{+}^{\rho}, & \rho>0, \\ \bar{F}_{\alpha}(0) \ln \frac{1}{\varepsilon}, & \rho=0 .\end{cases}
$$

Мы не рассматриваем в следствиях 1 и 2 случай $\alpha=1$, поскольку из условия (1.22) при $\alpha=1$ следует, что предположение (1.4) нарушается, а эта ситуация была детально изучена в [8].

Следствие 3. Пусть $\gamma>0, \rho \geqslant \alpha$. Если при некотором $\alpha \in(1,2]$ выполнень условия (1.4) и

$$
\frac{S_{n}}{n^{1 / \alpha}} \longrightarrow \xi_{\alpha} \quad \text { по вероятности при } \quad n \rightarrow \infty
$$

mo

$\sum_{n: \ln _{k} n \geqslant 1}\left(\ln _{k} n\right)^{\gamma \rho} \frac{1}{n \prod_{j=1}^{k} \ln _{j} n} \mathbf{P}\left\{S_{n} \geqslant \varepsilon n^{1 / \alpha}\left(\ln _{k} n\right)^{\gamma}\right\} \sim \frac{1}{\gamma \rho} \varepsilon^{-\rho} \mathbf{E}\left(\xi_{\alpha}\right)_{+}^{\rho}, \quad \varepsilon \searrow 0$,

тогда и только тогда, когда

$$
\mathbf{E I}\left[\ln _{k} X>0\right] X^{\alpha} \ln _{k}^{\gamma(\rho-\alpha)} X\left(\prod_{j=1}^{k} \ln _{j} X\right)^{-1}<\infty .
$$

Если $\alpha=2$ и $k \geqslant 2$ или $k=1$ и $\gamma(\rho-2) \leqslant 1$, то условие (1.25) вытекает из предположения

$$
\mathbf{E} X=0, \quad \mathbf{E} X^{2}=1 .
$$

Если же $k=1$ и $\gamma(\rho-2)>1$, то (1.25) равносильно условию $\mathbf{E} X_{+}^{2} \ln ^{\gamma(\rho-2)-1}\left(1+X_{+}\right)<$ $\infty$. Таким образом, следствие 3 обобщает и уточняет соответствуюшие результаты из [2], [9] и [4].

В заключение приведем еще одно следствие теоремы 1.

Следствие 4. Пусть $1<\alpha \leqslant 2,0<p<\alpha \leqslant r u \rho=\alpha(r-p) /(\alpha-p)$. Если выполнены условия (1.23) и (1.4), то

$$
\lim _{\varepsilon \searrow 0} \varepsilon^{\rho} \sum_{n \geqslant 1} n^{r / p-2} \mathbf{P}\left\{S_{n} \geqslant \varepsilon n^{1 / p}\right\}=\frac{p}{r-p} \mathbf{E}\left(\xi_{\alpha}\right)_{+}^{\rho}
$$

тогда и только тогда, когда $\mathbf{E} X_{+}^{r}<\infty$.

При $\alpha=2$ соотношение (1.27) является правосторонним вариантом (и уточнением) результата работы [6] (см. также [1]-[5]). Напоминаем, что из (1.23) при $1<\alpha \leqslant 2$ следует $\mathbf{E} X=0$, а если $\alpha=2$, то и $\mathbf{E} X^{2}<\infty$.

Проверка того, что следствия 1 и 2 вытекают из теоремы 2 , а следствия 3 и $4-$ из теоремы 1 , осуществлена в конце п. 3. 
2. Доказательство предложения 1. Обозначим $P_{n}(\varepsilon)=\mathbf{P}\left\{U_{n} \geqslant \varepsilon \varphi_{n}\right\}$. Пусть положительная функция $M=M(\varepsilon)$ как угодно медленно растет к бесконечности при $\varepsilon \searrow 0$. Положим $n_{1}=\max \left(n: \varphi_{n} \leqslant 1 / \varepsilon\right), n_{2}=\max \left(n: \varphi_{n} \leqslant M / \varepsilon\right)$. Имеем

$$
S(\varepsilon)=\left(\sum_{1 \leqslant n<M}+\sum_{M \leqslant n \leqslant n_{2}}+\sum_{n>n_{2}}\right) f_{n} P_{n}(\varepsilon)=S_{1}+S_{2}+S_{3} .
$$

Функция распределения $F_{\alpha}(x)$ абсолютно непрерывна и меньше единицы при любом конечном $x$, если $1 \leqslant \alpha \leqslant 2$. Отсюда и из условия (1.1) заключаем, что $\mathbf{P}\left\{U_{n} \geqslant x\right\} \rightarrow \bar{F}_{\alpha}(x)$ равномерно по $x$ при $n \rightarrow \infty$ и, следовательно,

$$
P_{n}(\varepsilon) \sim \bar{F}_{\alpha}\left(\varepsilon \varphi_{n}\right), \quad M \leqslant n \leqslant n_{2}, \quad \varepsilon \searrow 0,
$$

T.e.

$$
S_{2} \sim \sum_{M \leqslant n \leqslant n_{2}} f_{n} \bar{F}_{\alpha}\left(\varepsilon \varphi_{n}\right), \quad \varepsilon \searrow 0 .
$$

Учитывая, что $\psi_{\alpha}(\varepsilon) \nearrow \infty$ при $\varepsilon \searrow 0$, найдем

$$
\max \left(S_{1}, \sum_{1 \leqslant n<M} f_{n} \bar{F}_{\alpha}\left(\varepsilon \varphi_{n}\right)\right) \leqslant \sum_{1 \leqslant n<M} f_{n}=o\left(\psi_{\alpha}(\varepsilon)\right), \quad \varepsilon \searrow 0,
$$

если $M$ растет к бесконечности достаточно медленно.

Принимая во внимание, что в условиях предложения 1 правый хвост распределения $F_{\alpha}$ убывает быстрее любой степени $x$ (см., например, [11]), с помошью (1.5) при некоторых $\gamma, \delta>0$ получим

$$
\begin{aligned}
\sum_{n>n_{2}} f_{n} \bar{F}_{\alpha}\left(\varepsilon \varphi_{n}\right) & \leqslant A_{1} M^{-\gamma} \sum_{n>n_{1}} f_{n}\left(\varepsilon \varphi_{n}\right)^{-\delta} \leqslant A_{2} M^{-\gamma}\left(\varepsilon \varphi_{n_{1}}\right)^{-\delta} \sum_{n=1}^{n_{1}} f_{n} \\
& \leqslant \frac{A_{2} M^{-\gamma}\left(\varphi_{n_{1}+1} / \varphi_{n_{1}}\right)^{\delta}}{\bar{F}_{\alpha}(1)} \psi_{\alpha}(\varepsilon),
\end{aligned}
$$

где $A_{i}$ - некоторые постоянные, не зависящие от $\varepsilon$. Отсюда и из $(2.2),(2.3)$ следует

$$
S_{1}+S_{2} \sim \psi_{\alpha}(\varepsilon), \quad \varepsilon \searrow 0 .
$$

Сравнивая (2.1) и (2.5), видим, что соотношение (1.2) имеет место тогда и только тогда, когда

$$
S_{3}=o\left(\psi_{\alpha}(\varepsilon)\right), \quad \varepsilon \searrow 0 .
$$

Далее, покажем, что условие (1.7) является следствием (2.6).

Положим $D(x)=\mathbf{E} X^{2} \mathbf{I}[|X| \leqslant x]$.

Лемма 1 (см. [10, гл. XVII, §5], [13, гл. 4, §2]). Пусть выполняются условия предложения 1. Тогда функиия $D(x)$ правильно меняется на бесконечности с показателем $2-\alpha$,

$$
\frac{n D\left(B_{n}\right)}{B_{n}^{2}} \rightarrow c, \quad n a_{n}-\frac{n}{B_{n}} \int_{-B_{n}}^{B_{n}} x d F(x) \longrightarrow \gamma \quad n p u \quad n \rightarrow \infty,
$$

где с >0 и $\gamma$ - некоторые постоянные, $u$, кроме того, хвосты распределений $F$ и $F_{\alpha}$ удовлетворяют условиям $(x \rightarrow \infty)$

$$
\begin{aligned}
\frac{x^{2} \bar{F}(x)}{D(x)} & \rightarrow 0, & \frac{x^{2} F(-x)}{D(x)} & \rightarrow \frac{2-\alpha}{2}, \\
x^{\alpha} \bar{F}_{\alpha}(x) & \rightarrow 0, & x^{\alpha} F_{\alpha}(-x) & \rightarrow \frac{2-\alpha}{2} c .
\end{aligned}
$$

Лемма 2. Пусть $\left\{X_{n k} ; 1 \leqslant k \leqslant n, n \geqslant 1\right\}-$ последовательность серий независимьх и одинаково в каждой серии распределенных случайных величин. Положим $S_{n}=\sum_{k=1}^{n} X_{n k}, Q_{n}^{+}(u)=n \mathbf{P}\left\{X_{n 1} \geqslant u\right\}$. При любых $x$ и с имеет место оченка $\mathbf{P}\left\{S_{n}>x\right\} \geqslant(1-\eta) Q_{n}^{+}(c)$, , де $\eta=Q_{n}^{+}(c)+\mathbf{P}\left\{S_{n}-X_{n n}<x-c\right\}$. 
Утверждение леммы 2 следует из $[12,(33)]$.

Положим $X_{n k}=X_{k} / B_{n}-a_{n}, c=x-a_{n}, x=\varepsilon \varphi_{n}$ и $\varepsilon \varphi_{n} \geqslant 1$. С помошью лемм 1 и 2 получим, что при некотором $\delta>0$ и всех достаточно малых $\varepsilon>0$

$$
P_{n}(\varepsilon) \geqslant \delta \bar{F}\left(\varepsilon \varphi_{n} B_{n}\right) \text {. }
$$

Действительно, при $n \rightarrow \infty$, в силу $(2.7),(2.8)$, имеем $Q_{n}^{+}(c)=n \bar{F}\left(\varepsilon \varphi_{n} B_{n}\right) \leqslant$ $n \bar{F}\left(B_{n}\right)=o(1)$ и $1-\eta=\mathbf{P}\left\{S_{n}-X_{n} / B_{n} \geqslant 0\right\}+o(1)=\bar{F}_{\alpha}(0)+o(1) \geqslant \delta>0$.

Из (2.6) и (2.9) следует оценка

$$
\sum_{n \geqslant n_{2}} n f_{n} \bar{F}\left(\varepsilon b_{n}\right)=o\left(\psi_{\alpha}(\varepsilon)\right), \quad \varepsilon \searrow 0 .
$$

Кроме того (см. (2.7), (2.8)),

$$
\sum_{n=n_{1}}^{n_{2}} n f_{n} \bar{F}\left(\varepsilon b_{n}\right) \leqslant \frac{\max _{n \geqslant n_{1}} n \bar{F}\left(B_{n}\right)}{\bar{F}_{\alpha}(M)} \sum_{n=n_{1}}^{n_{2}} f_{n} \bar{F}_{\alpha}\left(\varepsilon \varphi_{n}\right)=o\left(\psi_{\alpha}(\varepsilon)\right), \quad \varepsilon \searrow 0,
$$

если $M$ растет достаточно медленно.

Итак, (1.7) есть следствие (2.6).

Докажем теперь, что из (1.5)-(1.7) вытекает (2.6).

Лемма 3. Положим (см. также обозначения в лемме 2)

$$
\Theta_{n}(u)=n \mathbf{P}\left\{X_{n 1}<u\right\}, \quad \beta_{2}(u)=u^{-2} \int_{-u}^{u} y^{2} d \Theta_{n}(y) .
$$

Пусть $\varkappa_{n}>0$ изменяется таким образом, ито

$$
\int_{|y|>\varkappa_{n}} d \Theta_{n}(y)+\beta_{2}\left(\varkappa_{n}\right)+\left|\frac{1}{\varkappa_{n}} \int_{-\varkappa_{n}}^{\varkappa_{n}} y d \Theta_{n}(y)\right| \longrightarrow 0, \quad n \rightarrow \infty .
$$

Тогда при любом $t>0$ и всех достаточно больших $n$ равномерно по $x>\max \left(\varkappa_{n}, 1\right)$

$$
\begin{aligned}
\mathbf{P}\left\{S_{n}>x\right\} \leqslant & Q_{n}^{+}\left(x-\varkappa_{n}\right)+Q_{n}^{+}\left(\frac{x}{3}\right)+\exp \left(-\frac{1}{3 \beta_{2}(x)}\right) \\
& +\exp \left(-\frac{x}{2}\right)+x^{-t} \int_{1}^{x} y^{t} d \Theta_{n}(y) .
\end{aligned}
$$

Лемма 3 представляет частный случай $\left[12,(4),(7),(9)\right.$ при $\gamma=\frac{1}{3}, \varepsilon=\frac{1}{2}, \lambda=1$, $r=1]$

Воспользуемся леммой 3 при $X_{n k}=X_{k} / B_{n}-a_{n}, x=\varepsilon \varphi_{n}$ и $n>n_{2}$ (см. (2.1)), предполагая, что $\varkappa_{n}=M / 2$, а $\varepsilon \searrow 0$. Поскольку в этом случае условие $(2.11)$ выполняется (см. соответствующие рассуждения в [8]), оценка (2.12) будет справедлива при всех достаточно малых положительных $\varepsilon$. Обозначим слагаемые в правой части неравенства $(2.12)$ через $I_{1}, \ldots, I_{5}$ соответственно и оценим их сверху.

При $x \geqslant M$, очевидно, $I_{1}=Q_{n}^{+}\left(x-\varkappa_{n}\right) \leqslant I_{2}=Q_{n}^{+}(x / 3) \leqslant n \bar{F}\left(x B_{n} / 4\right)$. Далее, $\int_{1}^{x} y^{t} d \Theta_{n}(y)=n \int_{\left(1+a_{n}\right) B_{n}}^{\left(x+a_{n}\right) B_{n}}\left(y / B_{n}-a_{n}\right)^{t} d F(y)$. Отсюда получим

$$
\int_{1}^{x} y^{t} d \Theta_{n}(y) \leqslant 2^{t}\left(n \int_{0}^{2 x B_{n}}\left(\frac{y}{B_{n}}\right)^{t} d F(y)+\left|a_{n}\right|^{t} n \bar{F}\left(\frac{B_{n}}{2}\right)\right) .
$$

Принимая во внимание лемму 1 , видим, что

$$
I_{1}+I_{2}+I_{4}+I_{5} \leqslant A x^{-t}\left(1+\frac{n}{B_{n}^{t}} \int_{0}^{x B_{n}} y^{t-1} \bar{F}(y) d y\right)
$$

где $A-$ некоторая положительная постоянная.

Теперь оценим слагаемое $I_{3}=\exp \left\{-1 /\left(3 \beta_{2}(x)\right)\right\}$. Имеем

$$
\beta_{2}(x)=\frac{n}{\left(x B_{n}\right)^{2}} \int_{\left(-x+a_{n}\right) B_{n}}^{\left(x+a_{n}\right) B_{n}}\left(y-a_{n} B_{n}\right)^{2} d F(y) .
$$


Следовательно, если $\mathbf{D} X=\sigma^{2}<\infty$, то равномерно по $x>x_{0}$

$$
\beta_{2}(x) \sim \frac{n \sigma^{2}}{\left(x B_{n}\right)^{2}}, \quad n \rightarrow \infty .
$$

Пусть теперь $\mathbf{D} X=\infty$. Поскольку $\left(y-a_{n} B_{n}\right)^{2} \geqslant \frac{1}{2} y^{2}-\left(a_{n} B_{n}\right)^{2}$, то применяя $(2.7)$ и оценку $\left(\int_{|y| \leqslant z}|y| d F(y)\right)^{2}=o(D(z)), z \rightarrow \infty$, найдем

$$
\begin{aligned}
& \beta_{2}(x) \geqslant \frac{n}{\left(x B_{n}\right)^{2}} \int_{|y| \leqslant x B_{n} / 2}\left(y-a_{n} B_{n}\right)^{2} d F(y) \geqslant \frac{n}{\left(x B_{n}\right)^{2}}\left(\frac{1}{2} D\left(\frac{B_{n}}{2}\right)-\left(a_{n} B_{n}\right)^{2}\right) ; \\
& \left(a_{n} B_{n}\right)^{2} \leqslant 2\left(\int_{|y| \leqslant B_{n}} y d F(y)\right)^{2}+2\left(\frac{B_{n}}{n}\right)^{2}(\gamma+o(1))^{2}=o\left(D\left(B_{n}\right)\right), \quad n \rightarrow \infty .
\end{aligned}
$$

Отсюда и из (2.14) следует, что

$$
\beta_{2}(x) \geqslant \frac{1}{3} \frac{n}{\left(x B_{n}\right)^{2}} D\left(\frac{x B_{n}}{2}\right) \geqslant x^{-\alpha+\eta}, \quad x>x_{\eta}, \quad \forall \eta>0,
$$

где последняя оценка имеет место в силу правильной изменчивости функции $D(z)$ (см. лемму 1).

Из $(2.12),(2.13)$ и $(2.15)$ при всех достаточно малых $\varepsilon$ и всех $n$ таких, что $\varepsilon \psi_{n}>M$, следует

$$
\mathbf{P}\left\{U_{n} \geqslant \varepsilon \psi_{n}\right\} \leqslant A\left(\left(\varepsilon \psi_{n}\right)^{-t}+\frac{n}{\left(\psi_{n} B_{n}\right)^{t}} \int_{0}^{\psi_{n} B_{n}} u^{t-1} \bar{F}(\varepsilon u) d u\right),
$$

где постоянная $t$ может быть сколь угодно велика, а $A$ не зависит от $n$ и $\varepsilon$.

Оценим $S_{3}$ по (2.16). Имеем

$$
S_{3} \leqslant A \sum_{n \geqslant n_{2}} f_{n}\left(\varepsilon \psi_{n}\right)^{-t}+A \sum_{n>n_{1}} f_{n} \frac{n}{\left(\psi_{n} B_{n}\right)^{t}} \int_{0}^{\psi_{n} B_{n}} u^{t-1} \bar{F}(\varepsilon u) d u=S_{31}+S_{32} .
$$

Здесь (см. (2.4))

$$
S_{31}=o\left(\psi_{\alpha}(\varepsilon)\right), \quad \varepsilon \searrow 0 .
$$

Остается оценить $S_{32}$. Положим $b_{n}=\psi_{n} B_{n}$. Очевидно,

$$
\sum_{n>n_{1}} n f_{n} b_{n}^{-t} \int_{0}^{b_{n}} u^{t-1} \bar{F}(\varepsilon u) d u \leqslant J_{1}+J_{2}
$$

где

$$
J_{1}=\int_{0}^{b_{n_{1}}} u^{t-1} \bar{F}(\varepsilon u) d u \sum_{n>n_{1}} n f_{n} b_{n}^{-t} .
$$

Имеем (см. (2.4)) при $\alpha<\delta \leqslant t$, обозначив $n_{1}+1$ через $m$ :

$$
\sum_{n>n_{1}} n f_{n} b_{n}^{-t} \leqslant A_{1} \frac{m \varphi_{m}^{\delta}}{b_{m}^{t}} \sum_{n>n_{1}} f_{n}\left(\varepsilon \varphi_{n}\right)^{-\delta} \leqslant A_{2} \frac{m}{b_{m}^{t}} \sum_{n=1}^{n_{1}} f_{n} \leqslant A_{3} \frac{m}{b_{m}^{t}} \psi_{\alpha}(\varepsilon) .
$$

Учитывая, что $\varepsilon \varphi_{m}>1$ (см. (2.1)) и $z^{-t} \int_{0}^{z} u^{t-1} \bar{F}(u) d u=o\left(D(z) / z^{2}\right), z \rightarrow \infty$ (см. лемму 1$)$, получим

$$
\begin{aligned}
\frac{m}{b_{m}^{t}} \int_{0}^{b_{m}} u^{t-1} \bar{F}(\varepsilon u) d u & \leqslant \frac{m}{B_{m}^{t}} \int_{0}^{B_{m}} u^{t-1} \bar{F}(u) d u \\
& =o\left(\frac{m D\left(B_{m}\right)}{B_{m}^{2}}\right)=o(1), \quad m \rightarrow \infty .
\end{aligned}
$$

Из (2.20)-(2.22) следует

$$
J_{1}=o\left(\psi_{\alpha}(\varepsilon)\right), \quad \varepsilon \searrow 0 .
$$


Рассмотрим величину $J_{2}$. Имеем

$$
\begin{aligned}
& J_{2}=\sum_{n>n_{1}} n f_{n} b_{n}^{-t} \sum_{k=n_{1}}^{n-1} \int_{b_{k}}^{b_{k+1}} u^{t-1} \bar{F}(\varepsilon u) d u \\
&=\sum_{k \geqslant n_{1}}\left(\sum_{n>k} n f_{n} b_{n}^{-t}\right) \int_{b_{k}}^{b_{k+1}} u^{t-1} \bar{F}(\varepsilon u) d u, \\
& \int_{b_{k}}^{b_{k+1}} u^{t-1} \bar{F}(\varepsilon u) d u \leqslant \bar{F}\left(\varepsilon b_{k}\right)\left(b_{k+1}-b_{k}\right) b_{k+1}^{t-1} .
\end{aligned}
$$

Принимая во внимание (см. (2.7)) соотношение $n / b_{n}^{2} \sim c /\left(\varphi_{n}^{2} D\left(B_{n}\right)\right), n \rightarrow \infty$, видим, что при всех достаточно больших $k$

$$
\sum_{n>k} n f_{n} b_{n}^{-t} \leqslant 2 \frac{k}{b_{k}^{2}} \sum_{n>k} f_{n} b_{n}^{-t+2}
$$

Отсюда, из (2.24) и из условий (1.5)-(1.7) найдем

$$
J_{2} \leqslant A \sum_{k \geqslant n_{1}} k f_{k} \bar{F}\left(\varepsilon b_{k}\right)=o\left(\psi_{\alpha}(\varepsilon)\right), \quad \varepsilon \searrow 0 .
$$

Вследствие (2.1), (2.16)-(2.19), (2.23) и (2.25), условие (2.6) вытекает из (1.7). Предложение 1 , таким образом, полностью доказано.

3. Прочие доказательства. Покажем, что теорема 1 вытекает из предложения 1. Нам следует проверить, что при условиях теоремы 1 имеют место соотношения (1.5), (1.6) и, кроме того,

$$
\psi_{\alpha}(\varepsilon) \sim \widetilde{\psi}_{\alpha}(\varepsilon), \quad \varepsilon \searrow 0 .
$$

Справедливость первого утверждения доказана в $[8$, п. 2].

Докажем, что соотношение (1.12) влечет $\left(1.6^{\prime}\right)$ и, следовательно, (1.6). Имеем в силу (1.11)

$$
A_{1} g\left(\varphi_{n+1}\right) \frac{\Delta \varphi_{n}}{\varphi_{n}} \leqslant f_{n} \leqslant A_{2} g\left(\varphi_{n}\right) \frac{\Delta \varphi_{n}}{\varphi_{n}}
$$

где постоянные $A_{i}$ зависят лишь от $\delta$. Отсюда, считая без потери общности, что постоянные $l, \delta, \gamma$ из условий $\left(1.6^{\prime}\right),(1.11),(1.12)$ удовлетворяют неравенству $l \geqslant \delta+\gamma$, и принимая во внимание условия (1.11), (1.12), найдем при $n>k$ и $k \rightarrow \infty$, что

$$
\begin{aligned}
f_{n} \frac{b_{n}^{-l}}{\Delta b_{n}} & \leqslant A_{2} \frac{\Delta \varphi_{n}}{\varphi_{n}} \frac{b_{n}}{\Delta b_{n}} b_{n}^{-\gamma} \frac{g\left(\varphi_{n}\right)}{b_{n}^{\delta}} b_{n}^{-l+\gamma+\delta-1} \leqslant A_{2} \frac{g\left(\varphi_{k+1}\right)}{b_{k+1}^{\delta}} b_{k+1}^{-l+\gamma+\delta-1} \frac{\Delta \varphi_{n}}{\varphi_{n}} \frac{b_{n}}{\Delta b_{n}} b_{n}^{-\gamma} \\
& =O(1) g\left(\varphi_{k+1}\right) \frac{\Delta \varphi_{k}}{\varphi_{k}} \frac{1}{\Delta b_{k}} b_{k}^{-l}\left(\frac{b_{k}}{b_{k+1}}\right)^{l+1-\gamma}=O(1) \frac{f_{k} b_{k}^{-l}}{\Delta b_{k}},
\end{aligned}
$$

что доказывает требуемое утверждение.

Проверим теперь справедливость соотношения (3.1). ІІусть положительная функция $M=M(\varepsilon)$ достаточно медленно растет к бесконечности при $\varepsilon \searrow 0$. Тогда (напоминаем, что $\left.\widetilde{\psi}_{\alpha}(0)=\infty\right)$

$$
\int_{1}^{M} g(u) \bar{F}_{\alpha}(\varepsilon u) \frac{d u}{u} \leqslant \int_{1}^{M} g(u) \frac{d u}{u}=o\left(\widetilde{\psi}_{\alpha}(\varepsilon)\right), \quad \varepsilon \searrow 0 .
$$

Далее (см. (2.4), (1.11)), если $l>\delta$, то

HO

$$
\begin{aligned}
\int_{M / \varepsilon}^{\infty} g(u) \bar{F}_{\alpha}(\varepsilon u) \frac{d u}{u} & \leqslant \varepsilon^{-l} \int_{M / \varepsilon}^{\infty} g(u) u^{-l} \frac{d u}{u} \leqslant \varepsilon^{-l} \frac{g(1 / \varepsilon)}{(1 / \varepsilon)^{\delta}} \int_{M / \varepsilon}^{\infty} u^{\delta-l} \frac{d u}{u} \\
& =o\left(g\left(-\frac{1}{\varepsilon}\right)\right), \quad \varepsilon \searrow 0,
\end{aligned}
$$

$$
\tilde{\psi}_{c}(\varepsilon) \geqslant \int_{1 / \varepsilon}^{2 / \varepsilon} g(u) \bar{F}_{\alpha}(\varepsilon u) \frac{d u}{u} \geqslant \bar{F}_{\alpha}(2) \frac{g(1 / \varepsilon)}{(1 / \varepsilon)^{\delta}} \int_{1 / \varepsilon}^{2 / \varepsilon} u^{\delta} \frac{d u}{u} \geqslant A g\left(\frac{1}{\varepsilon}\right)
$$


откуда следует, что

$$
\int_{M / \varepsilon}^{\infty} g(u) \bar{F}_{\alpha}(\varepsilon u) \frac{d u}{u}=o\left(\widetilde{\psi}_{\alpha}(\varepsilon)\right), \quad \varepsilon \searrow 0
$$

Из (3.2) и (3.3) вытекает

$$
\widetilde{\psi}_{\alpha}(\varepsilon) \sim \int_{M}^{M / \varepsilon} g(u) \bar{F}_{\alpha}(\varepsilon u) \frac{d u}{u}, \quad \varepsilon \searrow 0 .
$$

Аналогичные рассуждения приводят к равенству

$$
\widetilde{\psi}_{\alpha}(\varepsilon) \sim \sum_{n=n_{0}}^{n_{2}} \int_{\varphi_{n}}^{\varphi_{n+1}} g(u) \frac{d u}{u} \bar{F}_{\alpha}\left(\varepsilon \varphi_{n}\right)
$$

где $n_{0}=\max \left(n: \varphi_{n} \leqslant M\right), n_{2}=\max \left(n: \varphi_{n} \leqslant M / \varepsilon\right)$.

Соотношение (3.1) следует из (3.5) и (3.6), поскольку при условии $(1.8) \bar{F}_{\alpha}\left(\varepsilon \varphi_{n}\right) \sim$ $\bar{F}_{\alpha}\left(\varepsilon \varphi_{n+1}\right), n_{0} \leqslant n \leqslant n_{2}, \varepsilon \searrow 0$, если, конечно, $M$ растет к бесконечности не слишком быстро.

Далее, убедимся в справедливости замечания 2. Положим $h(x)=D(x) x^{\alpha-2}$ (см. лемму 1). При условиях (1.1), (1.4) функция $h(x)$ медленно меняется на бесконечности, $\bar{F}(z)=o\left(h(z) / z^{\alpha}\right), z \rightarrow \infty$, и $n h\left(B_{n}\right) / B_{n}^{\alpha} \rightarrow c$. Обозначим $\sum_{*} n f_{n} h\left(\varepsilon B_{n} \varphi_{n}\right)\left(\varepsilon B_{n} \varphi_{n}\right)^{-\alpha}$, где суммирование производится по $n$ таким, что $\varepsilon \varphi_{n} \geqslant 1$, через $J_{\varepsilon}$. Принимая во внимание вышесказанное, найдем

$$
\sum_{*} n f_{n} \bar{F}\left(\varepsilon B_{n} \varphi_{n}\right)=o\left(J_{\varepsilon}\right), \quad \varepsilon \searrow 0 .
$$

Пусть $0<\tau<\alpha-\delta$. Тогда, учитывая свойства медленно меняюшихся функций, получим

$$
J_{\varepsilon} \sim c \varepsilon^{-\alpha} \sum_{*} f_{n} \frac{h\left(\varepsilon \varphi_{n} B_{n}\right)}{h\left(B_{n}\right)\left(\varepsilon \varphi_{n}\right)^{\tau}} \varphi_{n}^{-\alpha}\left(\varepsilon \varphi_{n}\right)^{\tau} \leqslant 2 c \varepsilon^{\tau-\alpha} \sum_{*} f_{n} \varphi_{n}^{\tau-\alpha} \frac{h\left(\varepsilon \varphi_{n_{*}} B_{n_{*}}\right)}{h\left(B_{n_{*}}\right)\left(\varepsilon \varphi_{n_{*}}\right)^{\tau}},
$$

где $\varepsilon \varphi_{n_{*}} \geqslant 1>\varepsilon \varphi_{n_{*}-1}$. Отсюда и из $(1.8),(1.11)$ следует, что при $\varepsilon \searrow 0$

$$
J_{\varepsilon}=O(1) \varepsilon^{\tau-\alpha} \sum_{*} f_{n} \varphi_{n}^{\tau-\alpha}=O(1) \varepsilon^{\tau-\alpha} \int_{1 / \varepsilon}^{\infty} u^{\tau-\alpha} g(u) \frac{d u}{u}=O(1) g\left(\frac{1}{\varepsilon}\right) .
$$

Необходимый результат вытекает из $(3.7),(3.8)$ и (3.3).

Замечание 3 идентично [8, замечание 2 , пп. 1 и 2$]$.

Импликация $(1.17),(1.18) \Longrightarrow(1.12)$ несложно проверяется при помоши равенства

$$
\frac{\Delta b_{n}}{b_{n}}=\frac{B_{n+1}}{B_{n}} \frac{\Delta \varphi_{n}}{\varphi_{n}}+\frac{\Delta B_{n}}{B_{n}}
$$

с учетом соотношения (см. (2.7)) $B_{n+1} \sim B_{n}, n \rightarrow \infty$, и того, что $B_{n}$ имеет степенной порядок роста.

Утверждения относительно условий $(1.17),(1.18)$ вытекают из следуюшего элементарного результата.

\section{Лемма 4. Имеем}

$$
\liminf n \frac{\Delta \varphi_{n}}{\varphi_{n}}>0 \Longleftrightarrow \frac{\varphi_{n}}{n^{\gamma}} \nearrow, \quad \limsup n \frac{\Delta \varphi_{n}}{\varphi_{n}}<\infty \Longleftrightarrow \frac{\varphi_{n}}{n^{\gamma}} \searrow \quad(\exists \gamma>0) .
$$

Д ок а з а т е л с т в о. Проверим первое утверждение (второе проверяется аналогично). Положим $\bar{\varphi}_{n}=\varphi_{n} / n^{\gamma}, \gamma>0$. Тогда

и если $\bar{\varphi}_{n} \nearrow$, то

$$
\frac{\Delta \varphi_{n}}{\varphi_{n}}=\left(1+\frac{1}{n}\right)^{\gamma} \frac{\bar{\varphi}_{n+1}}{\bar{\varphi}_{n}-1}
$$

$$
n \frac{\Delta \varphi_{n}}{\varphi_{n}} \geqslant n\left(\left(1+\frac{1}{n}\right)^{\gamma}-1\right) \sim \gamma,
$$

откуда $\liminf n \Delta \varphi_{n} / \varphi_{n}>0$. 
Обратно, если $\Delta \varphi_{n} / \varphi_{n} \geqslant \delta>0$ при некотором $\delta$ и всех достаточно больших $n$, то при $0<\gamma<\min (1, \delta)$

$$
\begin{aligned}
\Delta \bar{\varphi}_{n} & =\frac{\Delta \varphi_{n}}{(n+1)^{\gamma}}-\left(\frac{1}{n^{\gamma}}-\frac{1}{(n+1)^{\gamma}}\right) \varphi_{n} \\
& \geqslant \frac{\delta \varphi_{n}}{n}(n+1)^{-\gamma}-(n+1)^{-\gamma} \varphi_{n}\left(\left(1+\frac{1}{n}\right)^{\gamma}-1\right) \geqslant \frac{(\delta-\gamma) \varphi_{n}}{n}(n+1)^{-\gamma}>0,
\end{aligned}
$$

т.е. $\bar{\varphi}_{n} \nearrow$. Лемма 4 доказана.

Равносильность условий (1.19) и (1.21) проверяется с помощью равенства

$$
\begin{aligned}
n \frac{\Delta B_{n}}{B_{n}} & =n \frac{\Delta h_{n}}{h_{n}}\left(1+\frac{1}{n}\right)^{1 / \alpha}+n\left(\left(1+\frac{1}{n}\right)^{1 / \alpha}-1\right) \\
& =(1+o(1)) n \frac{\Delta h_{n}}{h_{n}}+\frac{1}{\alpha}+o(1), \quad n \rightarrow \infty .
\end{aligned}
$$

Следствие 1 вытекает из теоремы 2 при $a_{n}=0$ (см. (1.1)) и $g(u)=u^{\rho}$, поскольку из $(1.21),(1.24)$ и $(3.9)$ следует, что $n \Delta \varphi_{n} / \varphi_{n} \rightarrow \gamma$ (напоминаем, что $\varphi_{n}=b_{n} / B_{n}$ ).

Следствие 2 аналогично [8, следствие 5] и также вытекает из теоремы 2.

Далее, докажем, что теорема 1 влечет следствие 3. Применим теорему 1 при $B_{n}=n^{1 / \alpha}, a_{n}=0, \varphi_{n}=\ln _{k}^{\gamma} n, g(u)=u^{\rho}, \rho \geqslant \alpha$. Учитывая, что

$$
\frac{\Delta \varphi_{n}}{\varphi_{n}} \sim \frac{\gamma}{n \prod_{j=1}^{k} \ln _{j} n}, \quad n \rightarrow \infty,
$$

найдем, что (1.24) равносильно условию

$$
\sum_{n: \varepsilon \varphi_{n} \geqslant 1} \frac{\ln _{k}^{\gamma \rho} n}{\prod_{j=1}^{k} \ln _{j} n} \bar{F}\left(\varepsilon n^{1 / \alpha} \ln _{k}^{\gamma} n\right)=o\left(\varepsilon^{-\rho}\right), \quad \varepsilon \searrow 0 .
$$

Стандартные рассуждения показывают, что (3.10) равносильно условию

$$
\int_{t_{\varepsilon}}^{\infty} t^{\alpha-1} \frac{\ln _{k}^{\gamma(\rho-\alpha)} t}{\prod_{j=1}^{k} \ln _{j} t} \bar{F}(t) d t=o\left(\left(\frac{1}{\varepsilon}\right)^{\rho-\alpha}\right), \quad \varepsilon \searrow 0
$$

в котором $t_{\varepsilon}=\left(\varphi^{-1}(1 / \varepsilon)\right)^{1 / \alpha}$, а $\varphi^{-1}(x)-$ функция, обратная к $\varphi(x)=\ln _{k} x$. Поскольку $\rho-\alpha \geqslant 0$, отсюда следует, что (3.10) равносильно (1.25), что и требовалось.

В заключение покажем, что следствие 4 вытекает из теоремы 1. Пусть в $(1.8),(1.9) \varphi_{n}=n^{\gamma}, g(u)=u^{\rho}$, где $\gamma=1 / p-1 / \alpha, p$ и $\rho$ такие, как в следствии 4. Если выполнены условия $(1.23),(1.4)$, то из теоремы 1 при $B_{n}=n^{1 / \alpha}, a_{n}=0$ (см. (1.1)) и соотношения (1.15) следует, что (1.27) равносильно условию

$$
I_{\varepsilon}=\sum_{n \geqslant \varepsilon^{-1 / \gamma}} n^{\gamma \rho} \bar{F}\left(\varepsilon n^{1 / p}\right)=o\left(\varepsilon^{-\rho}\right), \quad \varepsilon \searrow 0 .
$$

Несложно проверить, что (3.11) равносильно условию

$$
\int_{z}^{\infty} t^{r-1} \bar{F}(t) d t=o\left(z^{r-\alpha}\right), \quad z \rightarrow \infty
$$

которое, в свою очередь, равносильно соотношению $\mathbf{E} X_{+}^{r}<\infty$.

\section{СПИСОК ЛИТЕРАТУРЫ}

1. Baum L. E., Katz M. Convergence rates in the law of large numbers. - Trans. Amer. Math. Soc., 1965, v. 120, p. 108-123.

2. Gut A., Spătaru A. Precise asymptotics in the Baum-Katz and Davis laws of large numbers. - J. Math. Anal. Appl., 2000, v. 248, № 1, p. 233-246. 
3. Розовский Л.В. Некоторые предельные теоремы для больших уклонений сумм независимых случайных величин с общей функцией распределения из области притяжения нормального закона. -- Записки научн. семин. ПОМИ, 2002, т. 294, c. 165-193.

4. Li D. L., Wang X. C., Rao M. B. Some results on convergence rates for probabilities of moderate deviations for sums of random variables. - Internat. J. Math. Math. Sci., 1992, v. 15, № 3, p. 481-498.

5. Heyde C. C. A supplement to the strong law of large numbers. - J. Appl. Probab., 1975, v. 12, p. 173-175.

6. Chen $R$. A remark on the tail probability of a distribution. - J. Multivariate Anal., 1978 , v. 8, № 2, p. 328-333.

7. Spătaru A. Precise asymptotics in Spitzer's law of large numbers. - J. Theoret. Probab., 1999, v. 12, № 3, p. 811-819.

8. Розовский Л. В. О точной асимптотике в слабом законе больших чисел для сумм независимых случайных величин с общей функцией распределения из области притяжения устойчивого закона. - Теория вероятн. и ее примен., 2003, т. 48, B. 3 , с. $589-596$.

9. Gut A., Spătaru A. Precise asymptotics in the law of the iterated logarithm. - Ann. Probab., 2000, v. 28, № 4, p. 1870-1883.

10. Феллер B. Введение в теорию вероятностей и ее приложения. Т. 2. М.: Мир, 1984, 752 c.

11. Золотарев B. М. Одномерные устойчивые распределения. М.: Наука, 1983, 304 с.

12. Розовский Л. В. Одна оценка для вероятностей больших уклонений. - Матем. заметки, 1987, т. 42, № 1, с. 145-156.

13. Петров В.В. Предельные теоремы для сумм независимых случайных величин. M.: Наука, 1987,317 с.

Поступила в редакцию 5.II. 2003

(C) $2004 \Gamma$.

$$
\begin{gathered}
\text { ШоломицКИй А. } .^{*} \\
\text { О НЕОБХОДИМЫХ УСЛОВИЯХ } \\
\text { ПУАССОНОВСКОЙ СХОДИМОСТИ МАРТИНГАЛОВ }
\end{gathered}
$$

Доказана теорема о необходимых условиях пуассоновской сходимости сумм слагаемых, являющихся мартингал-разностями. Условия теоремы обобщают классические условия сходимости сумм независимых слагаемых.

Ключевье слова и фразы: мартингал, закон Пуассона, слабая сходимость.

B [6] были получены некоторые результаты о необходимых условиях нормальной сходимости сумм мартингал-разностей. Оказывается, что тем же методом можно получить необходимые условия и для пуассоновского случая.

Ниже символом $\mathscr{L}$ обозначается закон распределения случайной величины, сходимость законов понимается в смысле слабой сходимости соответствуюших распределений, пуассоновский закон с параметром $\lambda$ обозначается $\mathscr{P}(\lambda)$. Символы $\rightarrow$ и $\stackrel{\mathrm{P}}{\longrightarrow}$

* Центральный экономико-математический институт РАН, Нахимовский пр-т, 47 , 117418 Москва, Россия; e-mail: ags@cemi.rssi.ru

1) Работа частично поддержана грантом РФФИ 03-01-00479 и грантом РАН по 6-му конкурсу-экспертизе научных проектов молодых ученых (грант № 105). 